\title{
ON THE GROUP VELOCITY OF WHISTLING ATMOSPHERICS
}

\section{A.V. Guglielmi}

Schmidt Institute of Physics of the Earth RAS,

Moscow, Russia, guglielmi@mail.rutable

\section{B.I. Klain}

Borok Geophysical Observatory the Branch

of Schmidt Institute of Physics of the Earth RAS,

Borok, Russia,klb314@mail.ru

\author{
A.S. Potapov \\ Institute of Solar-Terrestrial Physics SB RAS, \\ Irkutsk,Russia,potapov@iszf.irk.ru
}

\begin{abstract}
The dynamic spectrum of a whistling atmospheric is a signal of falling tone, and the group delay time of the signal as a function of frequency is formed as a result of propagation of a broadband pulse in a medium (magnetospheric plasma) with a quadratic dispersion law. In this paper, we show that for quadratic dispersion the group velocity is invariant under Galilean transformations. This means that, contrary to expectations, the group velocity is paradoxically independent of the velocity of the medium relative to the observer. A general invariance condition is found in the form of a differential equation. To explain the paradox, we introduce the concept of the dynamic spectrum of Green's
\end{abstract}

function of the path of propagation of electromagnetic waves from a pulse source (lightning discharge in the case of a whistling atmospheric) in a dispersive medium. We emphasize the importance of taking into account the motion of plasma in the experimental and theoretical study of electromagnetic wave phenomena in near-Earth space.

Keywords: electromagnetic waves, moving plasma, Earth's magnetosphere, dispersion, propagation path, Green's function, dynamic spectrum.

\section{INTRODUCTION}

Lorentz invariance of the velocity of electromagnetic wave propagation in empty space is one of the tenets of the special relativity theory [Landau, Lifshitz, 1988]. In this case, group $v_{\mathrm{g}}$ and phase $v_{\mathrm{ph}}$ velocities are the same and both are equal to the universal constant - the speed of light $c=3 \cdot 10^{10} \mathrm{~cm} / \mathrm{s}$. The situation is different in a dispersive medium with a refractive index $n(\omega)$ : the group and phase velocities differ as

$$
v_{\mathrm{g}}=c / n_{\mathrm{g}}, \quad v_{\mathrm{ph}}=c / n,
$$

where $n_{\mathrm{g}}=\partial \omega n / \partial \omega$ is the so-called group refractive index; $\omega$ is the wave frequency. In this paper, we focus on another difference between the laws of electromagnetic wave propagation. This difference is radical and consists in the fact that during propagation in a material medium the group velocity changes when passing from one inertial frame of reference to another.

Let us introduce an accompanying frame of reference, in which a medium is static, and a laboratory frame moving relative to the medium uniformly and rectilinearly with a velocity $u$ in the direction of wave propagation. In the laboratory frame of reference, we designate physical quantities by primed symbols. We restrict ourselves to the nonrelativistic approximation $u<<c$ so that Lorentz transformations can be replaced by simpler Galilean transformations.

Common sense suggests that the group velocity obeys the usual law of velocity composition:

$$
v_{\mathrm{g}}=v_{\mathrm{g}}^{\prime}+u \text {. }
$$

In this paper, we draw attention to the fact that, contrary to common sense, in the case of whistling atmospherics, which are a type of geoelectromagnetic waves [Gershman, Ugarov, 1960; Guglielmi, Pokhotelov, 1996], the group velocity is invariant under Galilean transformations:

$$
v_{\mathrm{g}}=v_{\mathrm{g}}^{\prime} \text {. }
$$

On the one hand, this partly resembles the invariance of the wave propagation velocity in empty space. On the other hand, Formula (3) is a paradox that needs explanation.

In the next section, we find an invariance condition for the group velocity under Galilean transformations. Then, we briefly outline the theory of propagation of whistling atmospherics [Storey, 1953] and show that the group velocity of whistling atmospherics satisfies relation (3). We give examples of waves of other types whose group velocity is also invariant under Galilean transformations. To explain the paradox, we take into account properties and conditions of observation of whistling atmospherics and introduce the concept of the dynamic spectrum of Green's function of whistling atmospheric propagation path.

\section{INVARIANCE CONDITION}

Expand the group velocity in the laboratory frame of reference in a power series and restrict ourselves to the first two terms:

$$
v_{\mathrm{g}}^{\prime}\left(\omega^{\prime}\right)=v_{\mathrm{g}}^{\prime}(\omega)-\left.\frac{d v_{\mathrm{g}}^{\prime}}{d \omega^{\prime}}\right|_{\omega} k u .
$$


Here, we apply the Doppler formula $\omega=\omega^{\prime}+k u$. From (4) in view of the equality $v_{\mathrm{g}}^{\prime}\left(\omega^{\prime}\right)=v_{\mathrm{g}}(\omega)-u$, rewrite (3) as follows:

$$
\frac{d v_{\mathrm{g}}^{\prime}}{d \omega}=\frac{v_{\mathrm{ph}}^{\prime}}{\omega} .
$$

To avoid misunderstandings, it has to be said that here and below we consider the waves as slow $v_{\mathrm{ph}}<<c$ and, accordingly, neglect the well-known Fresnel correction to the propagation velocity (see, e.g., [Frankfurt, Frank, 1972]). For whistling atmospherics, the inequality $v_{\mathrm{ph}}<<c$ is satisfied with a large margin [Gershman, Ugarov, 1960].

Find the dispersion law $\omega(k)$ for waves whose group velocity remains unchanged when passing from the accompanying frame of reference to the laboratory one (see also [Guglielmi, 1963; Guglielmi, 1968]). To do this, represent equality (5) in the form of the following differential equation:

$$
f \frac{d^{2} f}{d \omega^{2}}+\left(\frac{d f}{d \omega}\right)^{2}=0,
$$

where $f(\omega)=\omega n(\omega)$. The equation has an analytical solution which gives

$$
\omega=a k^{2}+b,
$$

where $a$ and $b$ are arbitrary constants, with $a \neq 0$ on physical grounds. Thus, the equality $v_{\mathrm{g}}^{\prime}=v_{\mathrm{g}}$ holds provided that the frequency quadratically depends on the wavenumber.

\section{WHISTLING ATMOSPHERICS}

A whistling atmospheric is excited by a lightning discharge in the troposphere and, propagating in the magnetosphere along a geomagnetic field line, crosses the equatorial plane and reaches a conjugate point on Earth's surface in another hemisphere [Likhter et al., 1988]. When propagating, the pulse signal spreads out in such a way that at the output of a radio receiver we hear a whistle of a falling tone.

Note that the refractive index $n(\omega, \theta)$ in the magnetosphere depends on the angle $\theta$ between the wave vector $\mathbf{k}$ and the tangent to geomagnetic field line. The direction of the group velocity vector $\mathbf{v}_{\mathrm{g}}$ in the general case does not coincide with the direction of the $\mathbf{k}$ vector, and the value $v_{\mathrm{g}}=c / n_{\mathrm{g}}$ is equal to the projection of $\mathbf{v}_{\mathrm{g}}$ on the direction of $\mathbf{k}$ [Ginzburg, 1960].

In the geometrical optics approximation, the main laws of whistling atmospheric propagation are derived using the Storey formula:

$$
n=\frac{\omega_{0}}{\sqrt{\omega \Omega \cos \theta}} .
$$

Here, $\omega_{0}$ is the Langmuir plasma frequency; $\Omega$ is the electron gyrofrequency, $\omega<<\Omega, n>>1$ [Storey, 1953; Gershman, Ugarov, 1960]. Suppose that plasma moves along lines of the external magnetic field. This is a fairly realistic situation as there is a flow of plasma along geomagnetic field lines from the summer hemisphere to the winter one. From formula (8) it follows that

$$
\omega=\alpha k^{2},
$$

where $\alpha=\Omega k_{0}^{-2} \cos \theta ; k_{0}=\omega_{0} / c$, which coincides with (7) for $a=\alpha$ and $b=0$. Hence, the paradox $v_{\mathrm{g}}^{\prime}=v_{\mathrm{g}}$ arises because the dispersion relation for whistling atmospherics has the form of a quadratic dependence of the frequency on the wave number.

\section{EXPLANATION OF THE PARADOX}

It is quite clear that the measurement result depends on properties of an object under study and the instruments selected for the measurements. If the velocity of a narrowband wave train is measured with broadband receiving equipment, it is obvious that the velocity $v_{\mathrm{g}}^{\prime}=v_{\mathrm{g}}-u$. will be measured. The velocity $v_{\mathrm{g}}^{\prime}=v_{\mathrm{g}}$ will be measured when a broadband signal, excited by a pulse source, propagates along a sufficiently long trajectory in a medium with a quadratic dispersion law, and the receivers are selective, tuned to a fixed frequency.

There are two important clarifications to be made here. When describing the first experimental situation, we completely neglected the Fresnel correction. This is permissible only if the wave velocity is much lower than the velocity of light. The latter case requires a more detailed explanation. We can see that the paradox arises when a number of very special assumptions are made about properties of a signal and about the measurement method. However, whistling atmospherics have just these properties. As for measurements, broadband receivers are used for recording, but signal processing is performed by the method of spectral-temporal analysis with narrowband filtering as an essential element. Let us clarify what has been said by analyzing Green's function of the electromagnetic wave propagation path in magnetospheric plasma.

Let us make a brief excursus into foundations of the wave propagation theory. In the literature, the Cauchy problem is widely used such that at the initial moment in time $t=0$ the spatial structure of the wave field in the entire space is specified and it is required to find the space-time structure of the field at $t>0$. As an illustration, consider waves propagating in a positive direction of the $x$-axis in a homogeneous infinite medium. In the asymptotics, i.e. at large distances and at long times, an initial broadband pulse turns into a modulated quasisinusoidal wave. Its amplitude decreases with time as $1 / \sqrt{t}$. Spectral components of the initial pulse propagate along a family of world lines $x=v_{\mathrm{g}}(k) t$ in twodimensional space-time $(x, t)$. In other words, in the asymptotics there is an unfolded fan of space-time rays along which the spectrum of the initial pulse "spreads" (for more details, see the review [Vainshtein, 1976]).

It is easy to understand that the Cauchy problem does not in any way correspond to the conditions for excitation, propagation, and detection of whistling atmospherics. Here, it is appropriate to set the boundary value problem: a source is given at the point $x=0$ (in our case, it is a lightning discharge) and it is required to find 
a signal at the point of observation $x>0$. The segment [0, $x$ ] will be called the propagation path. In our case, the propagation path is a segment of the geomagnetic field line connecting a lightning discharge with a receiver. The receiver can be located on Earth's surface or in the ionosphere (on board a satellite). A delta-like pulse is fed to the input of the propagation path so that the solution to our boundary value problem

$$
G(x, t)=\frac{1}{2 \pi} \int_{-\infty}^{\infty} \exp [i k(\omega) x-i \omega t] d \omega
$$

is Green's function, or impulse response of the propagation path.

Finding Green's function is not easy even for a homogeneous path. The path length $x$ is fixed. We cannot therefore apply the concept of space-time rays $x / t=$ const, which approximately corresponds to the pattern of wave propagation when $t \rightarrow \infty, x \rightarrow \infty$. Instead of this we can, however, propose an asymptotic spectraltime representation of Green's function. The idea is as follows.

Determine the dynamic spectrum $\Gamma(\omega, t)$ of Green's function (10) by the following construction. Multiply the Fourier spectrum of Green's function by $2 \pi \exp \left[-\varepsilon(v-\omega)^{2}\right]$, i.e. cut out in the spectrum a window of width $\varepsilon^{-1 / 2}$ centered at the frequency $v=\omega$. Then, perform the inverse Fourier transform:

$$
\begin{aligned}
& \Gamma(\omega, t)= \\
& =\int_{-\infty}^{\infty} \exp \left[-i v t+i \frac{x}{c} v n(v)-\varepsilon(v-\omega)^{2}\right] d v .
\end{aligned}
$$

For a sufficiently large $\varepsilon$, the main contribution to the integral is made by the frequencies $v$ close to $\omega$. Introduce the notation $\xi=v-\omega$ and expand $n(v)$ in a power series of $\xi$ up to a quadratic term. This yields

$$
\begin{aligned}
& \Gamma(\omega, t)=\int_{-\infty}^{\infty} d \xi \exp \left\{i \xi\left[\frac{x}{c} n_{\mathrm{g}}(\omega)-t\right]+\right. \\
& \left.+\frac{\xi^{2}}{2}\left[i \frac{x}{c} \frac{d n_{\mathrm{g}}}{d \omega}-\varepsilon\right]\right\},
\end{aligned}
$$

or after integration

$$
\Gamma(\omega, t)=\sqrt{\frac{2 \pi}{\varepsilon-i \tau^{\prime}(\omega)}} \exp \left\{-\frac{[t-\tau(\omega)]^{2}}{2\left[\varepsilon-i \tau^{\prime}(\omega)\right]}\right\} .
$$

Here, $\tau=(x / c) n_{\mathrm{g}}, \tau^{\prime}=d \tau / d \omega$. Obviously, $|\Gamma(\omega, t)|$ peaks at $t=\tau(\omega)$. The quantity $\tau(\omega)$ is called the group delay time.

It remains to use Storey formula (8) to calculate the group delay time of whistling atmospherics:

$$
\tau(\omega)=\frac{x}{2 \sqrt{\alpha \omega}} .
$$

Since $\tau=x / v_{\mathrm{g}}$ and hence $v_{\mathrm{g}}=v_{\mathrm{g}}^{\prime}=2(\alpha \omega)^{1 / 2}$, we infer that the paradox becomes clear. Formula (3) is valid in its domain of applicability. However, we should not overlook wave field properties and specific conditions for observing and analyzing whistling atmospherics.

\section{DISCUSSION}

Lightning discharges excite not only whistling atmospherics, but also so-called spherics [Guglielmi, Pokhotelov, 1996]. Unlike whistling atmospheric, a spheric propagates in the Earth-ionosphere waveguide, not in the magnetosphere. Condition (7) holds at frequencies close to the critical frequency of the waveguide, i.e. the frequency quadratically depends on the wave number.

Langmuir waves are a classic example of waves with a quadratic dispersion law. In collisionless plasma, Langmuir waves experience Landau damping [Pitaevsky, Lifshitz, 1979]. We ignore the damping. Then the dispersion relation takes the form

$$
\omega=\omega_{0}\left(1+\frac{3}{2} D^{2} k^{2}\right),
$$

where $D$ is the Debye radius [Kadomtsev, 1968].

An interesting example of waves with the quadratic dispersion law is provided by quantum mechanics. It follows from the Schrödinger equation that the dispersion law for a free electron has the form

$$
\omega=\frac{\hbar}{2 m} k^{2},
$$

where $m$ is the electron mass, $\hbar$ is the Planck constant [Landau, Lifshitz, 1989]. In the form, (16) coincides with dispersion relation (10) for whistlers so that formally $v_{\mathrm{g}}^{\prime}=v_{\mathrm{g}}$.

Discuss briefly the issue of taking into account the longitudinal inhomogeneity of the propagation path when calculating the group delay time of a whistling atmospheric. The longitudinal inhomogeneity can be easily taken into account by the geometrical optics method [Ginzburg, 1960]. As a result, instead of (14) we have

$$
\tau(\omega)=\frac{1}{2 \sqrt{\omega}} \int_{0}^{x} \frac{d x}{\sqrt{\alpha(x)}} .
$$

An extensive literature is devoted to considering the magnetospheric plasma inhomogeneity in the analysis of whistling atmospherics, which is reflected in many reviews and monographs (see, e.g., [Likhter et al., 1988]).

At the end of this section, we would like to emphasize that in the work we have used basic physical concepts of space, time, frame of reference, electromagnetic field, dispersive medium and have correctly performed standard mathematical calculations. Nevertheless, the result obtained may seem contrary to the practice of observing electromagnetic waves, but only at first glance. A whistling atmospheric is a vivid example of an electromagnetic signal propagating with a group velocity independent of the velocity of the medium along the propagation path.

The result, which is interesting in itself, has certain methodological significance. It is well known that a whistler can repeatedly go along the same path, being reflected from the ionosphere at magnetoconjugate points. Place two identical broadband radio receivers at conjugated points. It would seem that the difference in 
travel times of a signal from the Northern Hemisphere to the Southern Hemisphere and back (or vice versa) can be used to estimate the velocity of travel of magnetospheric plasma along geomagnetic field lines. Our result suggests that such a possibility is excluded. We should, however, make a minor reservation. What is meant here, are typical whistlers, which are signals of a falling tone. Signals of a special type, aka nose whistlers, are in principle suitable for diagnosing the plasma velocity, but this is a topic for another work.

\section{CONCLUSION}

The influence of plasma motion on the nature of the observable electromagnetic wave phenomena is of undoubted interest for magnetospheric physics. We drew attention to the unusual property of whistling atmospherics, expressed by Formula (3). It is not so easy to interpret the formula as it might seem. We had to take into account the features of propagation of broadband signals, excited by a pulse source, in a medium with a quadratic dispersion law, as well as the conditions for recording and processing signals. We have introduced the concept of the dynamic spectrum of Green's function for the propagation path of a whistling atmospheric. The result of the analysis suggests that the frequency dependence of the group delay of a whistling atmospheric is not affected by the velocity of travel of magnetospheric plasma relative to the observer.

We express our sincere gratitude to G.A. Zherebtsov for his attention to our work and valuable advice. We thank O.D. Zotov and F.Z. Feigin for stimulating discussions. We are grateful to the reviewers for thorough analysis of our manuscript.

This work was financially supported by the Russian Foundation for Basic Research (project No. 19-0500574), as well as by programs of government assignments of IPE RAS and ISTP SB RAS.

\section{REFERENCES}

Frankfurt U.I., Frank A.M. Optika dvizhushchikhcya tel [Optics of Moving Bodies]. Moscow, Nauka Publ., 1972, 212 p. (In Russian).

Gershman B.N., Ugarov V.A. Propagation and generation of low-frequency electromagnetic waves in the upper atmosphere. Soviet Physics Uspekhi. 1961, vol. 3, pp. 743-764. DOI: 10.1070/PU1961v003n05ABEH005809.
Ginzburg V.L. Rasprostranenie elektromagnitnykh voln v plazme [The Propagation of Electromagnetic Waves in Plasmas]. Moscow, Fizmatgiz Publ., 1960, 552 p. (In Russian). (English edition: Ginzburg V.L. The Propagation of Electromagnetic Waves in Plasmas. Translated from the Russian edition (Moscow, 1960) by J.B. Sykes and R.J. Tayler. Pergamon Press, 1964, 535 p.)

Guglielmi A.V. On the group velocity of slow waves in a drifting magnetoactive plasma. Geomagnetism and Aeronomy. 1963, vol. 3, no. 4, pp. 754-757. (In Russian).

Guglielmi A.V. The propagation of slow waves in a moving plasma. Ann. Geophys. 1968, vol. 24, no. 3, pp. 761-763.

Guglielmi A.V., Pokhotelov O.A. Geoelectromagnetic waves. Bristol and Philadelphia, IOP Publ. Ltd., 1996, 402 p.

Kadomtsev B.B. Landau damping and echo in a plasma. Soviet Physics Uspekhi. 1968, vol. 11, pp. 328-337.

Landau L.D., Lifshitz E.M. Teoriya polya [Field Theory]. Moscow, Nauka Publ., 1988, 512 p. (In Russian). (English edition: Landau L.D., Lifshitz E.M. The Classical Theory of Fields. Pergamon Press, 1975, 403 p.)

Landau L.D., Lifshitz E.M. Kvantovaya mekhanika [Quantum Mechanics]. Moscow, Nauka Publ., 1989, 768 p. (In Russian). (English edition: Landau L.D., Lifshitz E.M. Quantum Mechanics. Non-Relativistic Theory. Pergamon Press, 1977, 674 p.).

Likhter Ya.I., Guglielmi A.V., Erukhimov L.M., Mikhailova G.A. Volnovaya diagnostika prizemnoi plazmy [Wave diagnostics of surface plasma]. Moscow, Nauka Publ., 1988, 216 p. (In Russian).

Pitaevsky L.P., Lifshitz E.M. Fizicheskaya kinetika [Physical Kinetics] Moscow, Nauka Publ., 1979, 807 p. (In Russian). (English edition: Pitaevsky L.P., Lifshitz E.M. Physical Kinetics. Butterworth-Heinemann, 1981, 625 p.).

Storey L.R.O. An investigation of whistling atmospherics. Philosophical Transactions of the Royal Society. 1953, vol. 246, no. 908, pp. 113-141. DOI: 10.1098/rsta.1953.0011.

Vainshtein L.A. Propagation of pulses. Soviet Physics Uspekhi. 1976, vol. 19, pp. 189-205. DOI: 10.3367/UFNr. 0118.197602h.0339.

How to cite this article

Guglielmi A.V., Klain B.I., Potapov A.S. On the group velocity of whistling atmospherics. Solar-Terrestrial Physics. 2021. Vol. 7. Iss. 4. P. 67-70. DOI: $10.12737 /$ stp-74202106. 